\title{
Grid-Free Particle Method for the Inhomogeneous Enskog Equation and its Application to a RIEMANN-PROBLEM
}

\author{
Lars Popken \\ Fachbereich Mathematik \\ Universität Kaiserslautern \\ 67653 Kaiserslautern, Germany \\ e-mail: popken@mathematik.uni-kl.de
}

\begin{abstract}
Starting from the mollified version of the Enskog equation for a hard-sphere fluid, a grid-free algorithm to obtain the solution is proposed. The algorithm is based on the finite pointset method. For illustration, it is applied to a Riemann problem. The shock-wave solution is compared to the results of Frezzotti and Sgarra where a good agreement is found.
\end{abstract}

Key Words: Dense gas, Enskog equation, finite pointset method, kinetic theory, particle method, shock wave.

\section{Introduction}

A kinetic equation describing the behaviour of moderately dense gases was first proposed by Enskog [5] 1921 for the single-component distribution function $f: \mathbb{R}_{+} \times \Omega \times \mathbb{R}^{3} \rightarrow \mathbb{R}_{+}$:

$$
\frac{\partial f}{\partial t}+v \cdot \nabla_{x} f=J_{E}(f)
$$


and

$$
\begin{array}{r}
J_{E}(f)=\iint_{\mathbb{R}^{3} \times S^{2}} k\left(v_{21} \cdot \eta\right) \\
\left.-g^{(2)}(t, x, x-a \eta) f(t, x, v) f\left(t, x-a \eta, v_{*}\right)\right] d \eta d v_{*}
\end{array}
$$

In Eq. (1), $a$ is the diameter of the molecules, $v_{21}=v_{*}-v$ is the relative speed of the centers, $\eta$ is the unit vector from the center of the second sphere (*-indexed) to the center of the first, $k\left(v_{21} \cdot \eta\right)=a^{2}\left(v_{21} \cdot \eta\right) \theta\left(v_{21} \cdot \eta\right)$ for hard spheres, where $\theta$ is the Heaviside function. The primed variables denote the postcollisional values:

$$
v^{\prime}=v+\left(v_{21} \cdot \eta\right) \eta, \quad v_{*}^{\prime}=v_{*}-\left(v_{21} \cdot \eta\right) \eta
$$

$g^{(2)}$ is the pair-correlation function which accounts for spatial correlations. In the context of the revised Enskog theory $(\mathrm{RET}), g^{(2)}=g^{(2)}\left(x, x_{*} \mid n(t)\right)$ is the local equilibrium pair-correlation function expressed as a functional of the number density $n(t)$. For further detail refer to Résibois [14].

In the framework of the so called Standard Enskog Theory (SET), $g^{(2)}$ is simply set equal to the value of the equilibrium pair-correlation function at contact, evaluated as a function of the uniform density $n$ at the point of contact, see e.g. Carnahan \& Starling [4], where $g^{(2)}=g^{(2)}(n(x))$.

In this work we use the approximation suggested by Goldshtein, Shapiro and Gutfinger [8] for $g^{(2) \text { : }}$

$$
g^{(2)}(\nu)=\left[1-\left(\nu / \nu_{M}\right)^{4 \nu_{M} / 3}\right]^{-1}
$$

where $\nu=\frac{b n}{4}$ is the reduced density, $b=\frac{2}{3} \pi a^{3}$ and $\nu_{M}$ is the maximal packing density. Approximation (3) is accurate for both the low-density (see Carnahan \& Starling) and the high- density (see Alder \& Hoover [1]) limiting situations. In contrast to the Boltzmann collision integral, the molecules are now equipped with a diameter $a>0$ and thus the collision partners are at different positions. In the limit $\nu \rightarrow 0$, the pair-correlation $g^{(2)} \rightarrow 1$ and (1) recovers the Boltzmann collision integral for hard-spheres.

Up to now, only few attempts have been made to solve numerically the Enskog equation by kinetic schemes. Frezzotti \& Sgarra [7] combine a finite difference scheme with Monte Carlo techniques to evaluate the collision integral and apply it to a Riemann-problem for slightly dense gases. A Nanbu-like particle scheme for the Boltzmann equation (BE) [12] has been modified to solve the Enskog equation by Montanero \& Santos [9]. They apply their Enskog simulation Monte Carlo (ESMC) method to a uniform 
shear flow. Yet, their approach conserves energy and momentum only in a statistical sense and not in a single collision.

Very recently, Frezzotti [6] extended Bird's DSMC method [3] to calculate the density profile of a dense gas in equilibrium near a hard wall.

In this paper a grid-free particle method is presented, which is an extension of Nanbu's version as well. Care is taken that every single collision conserves momentum and energy and are thus conserved globally. It is based on the mathematically sound finite pointset method (FPM), which is sustained by the weak formulation of the discretised Enskog equation.

The organisation of the paper is as follows: At first, a measure formulation for the mollified time-discrete Enskog equation is derived in Section 2. In Section 3., measure approximation leads to an algorithm (particle method) for the solution of (EE). As an illustration, the method is applied to a Riemann problem in Section 4, where the formation of shock-profiles can be observed. The shock wave solutions are compared to Frezzotti's results. Finally, the results are discussed in Section 5.

\section{Measure Formulation for the Time- Discrete Mollified Enskog Equation}

With the help of the one-particle distribution $f(t, x, v)$ the macroscopic moments of $f$ are obtained, e.g. the number density $n$, the mean velocity $u$ or the temperature $T$

$$
\begin{aligned}
n(t, x) & =\int f(t, x, v) d v \\
u(t, x) & =(n(t, x))^{-1} \int v f(t, x, v) d v \\
T(t, x) & =(3 n(t, x) R)^{-1} \int|v-u(t, x)|^{2} f(t, x, v) d v
\end{aligned}
$$

where $R$ is the gas constant.

Similar to the numerical approach to the full Boltzmann equation, a time splitting of (EE) into a free flow and a collision term is established:

For this, a time step $\Delta t>0$ is chosen, $t_{k}=k \Delta t$, and $f^{k}=f\left(t_{k}, x, \tau\right)$, $k=0,1, \ldots$ The splitting scheme is then

$$
\begin{aligned}
& \frac{\partial \bar{f}^{k}}{\partial t}=-v \cdot \nabla_{x} f^{k}, \\
& \frac{\partial f^{k}}{\partial t}=J_{E}\left(\bar{f}^{k}\right) .
\end{aligned}
$$


As it is well-known how to handle the free-flow part (7), we concentrate on the non-homogeneous collisional part (8), which, for simplicity, is written as $\frac{\partial f^{k}}{\partial t}=J_{E}\left(f^{k}\right)$ throughout the rest of this section.

Now let $\Omega \subset \mathbb{R}^{3}$ be the domain of position and $[0, T]$ the time-domain. Then let

$$
N_{\Omega}^{t}:=\int_{\Omega} n(t, x) d x<\infty
$$

be the number of molecules in $\Omega$. The time-discrete formulation of (8) over a time-step $\Delta t$ is then

$$
f(t+\Delta t, x, \tau)=f(t, x, \tau)+\Delta t J_{E}(f(t, ., .))
$$

Let $\varphi: \Omega \times \mathbb{R}^{3} \rightarrow \mathbb{R}$ be a continuous test function. Multiplication of (9) and integration over the phase-space $\Omega \times \mathbb{R}^{3}$-space leads to

$$
\begin{array}{r}
\iint_{\Omega \times \mathbb{R}^{3}} \varphi(x, v) f(t+\Delta t, x, v) d v d x=\iint_{\Omega \times \mathbb{R}^{3}} \varphi(x, v) f(t, x, v) d v d x+ \\
+\Delta t \iiint_{\Omega \times \mathbb{R}^{3} \times \mathbb{R}^{3} \times S^{2}} k\left(v_{21} \cdot \eta\right) g^{(2)}\left(n\left(t, x-\frac{a}{2} \eta\right)\right)\left[\varphi\left(x, v^{\prime}\right)-\varphi(x, v)\right] \times \\
\times \quad f(t, x, v) f\left(t, x-a \eta, v_{*}\right) d \eta d v_{*} d v d x
\end{array}
$$

A usual approach to (8) in the rarefied gas regime is the projection of the initial density $f(t, . .$.$) onto locally constant functions on an arbitrary parti-$ tion of $\Omega$ and the application of a particle method working independently on each of these cells (homogenisation). This method is no more suitable now, as for each collision $f$ has to be evaluated at different positions of a distance near to or much bigger than the mean free path, i.e. particles from different cells might interact and the advantage of local homogenisation has vanished.

A suitable approach to handle the evaluation of the distribution function at different positions is found by the discretisation of the collision operator in the spatial domain with the help of a mollifier. The idea of smoothing the Boltzmann equation has been introduced by Morgenstern [10] and extended by Steiner [15] who derived numerical schemes for (BE) using mollifiers. This ansatz is now applied to the Enskog equation, which changes the 
collision operator $J_{E}(f)$ in (1) to

$$
\begin{aligned}
J_{E}^{\beta}(f)= & \iiint_{\mathbb{R}^{3} \times \mathbb{R}^{3} \times S^{2}} k\left(v_{21} \cdot \eta\right) \beta_{h}\left(x, x_{*}\right)\left[g^{(2)}\left(n\left(t, \frac{1}{2}\left(x+x_{*}+a \eta\right)\right)\right) f\left(t, x, v^{\prime}\right) f\left(t, x_{*}+a \eta, v_{*}^{\prime}\right)\right. \\
& \left.-g^{(2)}\left(n\left(t, \frac{1}{2}\left(x+x_{*}-a \eta\right)\right)\right) f(t, x, v) f\left(t, x_{*}-a \eta, v_{*}\right)\right] d \eta d v_{*} d x_{*}
\end{aligned}
$$

where $\beta_{h}(x,$.$) is for any x$ a mollifier with compact support $\Omega_{\beta}(x)$. The mollifier has to fulfill certain conditions like symmetry, normalization and boundedness such that $J_{E}^{\beta} \rightarrow J_{E}$ if $\beta_{h}(x,$.$) converges to Dirac's \delta$-function.

A change of variable $\tilde{x}_{*}=x_{*}-a \eta$ and the introduction of measures $d \mu^{t}=f(t, x, v) d v d x$ and $d \mu_{*}^{t}=f\left(t, \tilde{x}_{*}, v_{*}\right) d \tau_{*} d \tilde{x}_{*}$ delivers the time-discretised measure-valued Enskog equation (without free flow term):

$$
\begin{aligned}
\iint_{\Omega \times \mathbb{R}^{3}} \varphi(x, v) d \mu^{t+\Delta t}= & \int \ldots \int_{S^{2} \times \Omega \times \mathbb{R}^{3} \times \Omega_{\beta}^{\eta}(x) \times \mathbb{R}^{3} \times[0,1]} \frac{1}{4 \pi N_{\Omega_{\beta}^{\eta}(x)}^{t}}\left(\varphi\left(x, v^{\prime}\right) \mathcal{X}_{\left[0, \Delta t \tilde{q}_{21}\right]}(\kappa)+\right. \\
& \left.+\varphi(x, v) \mathcal{X}_{\left[\Delta t \tilde{q}_{21}, 1\right]}(\kappa)\right) d \kappa d \mu_{*}^{t} d \mu^{t} d \eta
\end{aligned}
$$

The idea of representing the weak formulation of the collision integral via a characteristic function $\mathcal{X}$ is due to Struckmeier [16, 13].

In equation (13), $\Omega_{\beta}^{\eta}(x)=\left\{y \mid y+a \eta \in \Omega_{\beta}(x)\right\}$ and

$$
\tilde{q}_{21}=4 \pi N_{\Omega_{\beta}^{\eta}(x)}^{t} k\left(v_{21} \cdot \eta\right) \beta_{h}\left(x, \tilde{x}_{*}+a \eta\right) g^{(2)}\left(n\left(t, \frac{1}{2}\left(x+\tilde{x}_{*}\right)\right)\right)
$$

is the collision probability for two particles.

Equation (13) renders the measure of $\varphi$ with respect to the density $f$ at time $t+\Delta t$ as a function of the measure $\varphi$ at time $t$ with respect to a product of density functions, i.e. if the measures $\mu$ and $\mu_{*}$ are known at time $t$, then the measure $\mu$ at time $t+\Delta t$ is computable via (13).

Equation (13) is reasonable in the sense of measures, i.e. non-negativity of $f(t+\Delta t)$ is guaranteed for non-negative $f(t)$, if

$$
\Delta t \tilde{q}_{21} \leq 1 \quad \forall \text { admissible }\left(x, \tilde{x}_{*}, \eta, v_{21} \cdot \eta\right) .
$$

This is a restriction for the time discretisation $\Delta t$ in the Euler scheme! 


\section{Remark 2.1.}

1. If $\bigcup_{k \in \mathbb{K}} Z_{k}^{h}=\Omega$ is a disjoint partition of $\Omega$ with $\operatorname{diameter} \operatorname{diam}\left(Z_{k}^{h}\right)=$ $\sup \left\{\left\|x-x_{*}\right\|, x, x_{*} \in Z_{k}^{h}\right\} \leq h$ for all $k \in \mathbb{K}$ then

$$
\beta_{h}^{h o m}\left(x, x_{*}+a \eta\right)=\frac{\sum_{k} \mathcal{X}_{Z_{k}^{h}}(x) \mathcal{X}_{Z_{k^{*}}^{h}}\left(x_{*}\right)}{\sum_{k} \mathcal{X}_{Z_{k}^{h}}(x) \cdot \operatorname{meas}\left(Z_{k^{*}}^{h}\right)}
$$

is a grid-inducing mollifier allowing collisions of particles of different cells. A mollifier of this kind where $Z_{k}^{h}$ are cubic cells of (constant) length $h$ has been used by Montanero et al. Here, $h$ has to be less than the diameter $a$ of the molecules, such that for a particle at position $x$ in cell $Z_{k}^{h}$ and a collision direction $\eta$, a possible collision partner is in general in a different cell $Z_{k^{*}}^{h}$, containing the position $x-a \eta$.

2. In this work, a local mollifier is used that is no more based on a grid:

$$
\beta_{h}^{l o c}\left(x, x_{*}+a \eta\right)=\beta_{h}^{l o c}(y)=\frac{1}{h} \mathcal{X}_{[-h / 2, h / 2]}(y), \quad y=x-x_{*}-a \eta,
$$

where the mean free path $\lambda=\left(\sqrt{2} \pi n a^{3} g^{(2)}(n)\right)^{-1}$ is taken as the maximum value for $h: h \leq \lambda$. The mollifier $\beta_{h}^{\text {loc }}$ allows a particle at position $x$ to find a collision partner in a mean free path environment of $x-a \eta$ with no regard for a grid. With the above mollifier the attempt to locally homogenise the domain is no more necessary.

\section{Particle Method for the Enskog Equation}

In this section the principles of particle approximations are introduced and applied to (13). This delivers an algorithm for the numerical solution of one time step.

\subsection{Particle Approximations}

A particle is characterized by its position $x$, velocity $v$ and mass $\alpha$. A particle set (or finite point set) is given by

$$
\mu_{N}=\left\{\left(\alpha_{1}, x_{1}, v_{1}\right), \ldots,\left(\alpha_{N}, x_{N}, v_{N}\right)\right\}
$$

or - in different notation - by

$$
\delta_{\mu_{N}}=\sum_{i=1}^{N} \alpha_{i} \delta_{x_{i}} \delta_{v_{i}}
$$


Now, for a given density $f \in \mathcal{L}_{+}^{1}$ we say that ' $\delta_{\mu_{N}}$ converges to $f$ ', if

$$
\lim _{N \rightarrow \infty} \sum_{i=1}^{N} \alpha_{i} \varphi\left(x_{i}, v_{i}\right)=\int f \cdot \varphi d v d x, \quad \text { for all } \varphi \in C^{b}\left(\mathbb{R}^{3} \times \mathbb{R}^{3}\right) .
$$

This means that the discrete measure $\delta_{\mu_{N}}$ converges weakly* to $f d v d x$. Eq. (20) can be interpreted as an integration rule, where we integrate the function $\varphi$ with respect to the measure $f d v d x$. The idea of particle methods is now to approximate continuous measures by discrete ones with the help of (20). Approximating the right-hand side of eq. (13) with respect to some distance between $\mu_{N}$ and $f$ (discrepancy) thus leads to a finite pointset method for the approximation of the distribution function $f$ at time $t+\Delta t$.

Remark 3.1. Further detail to the approximation of continuous measures by finite point sets and the quality of approximation can be found in Struckmeier [16]. He also states how to include unbounded $\varphi$ such as $|v|^{2}$ or $|v|^{2} v$ etc.

\subsection{Finite Pointset Method for the Inhomogeneous Enskog Equation}

Concerning the discretisation of the inhomogeneous equation we use a decoupling of the free flow of particles and the collisions among them (splitting scheme (7) \& (8)).

Free Flow: Given an approximation of $f(k \Delta t, x, v)$ by a finite pointset we use (7) to obtain

$$
\bar{f}((k+1) \Delta t, x, v)=f(k \Delta t, x-\Delta t v, v),
$$

i.e. we just move the particles over the time increment $\Delta t$ with the particle velocity and no spatial discretisation is required.

Collision Term: Let $\mu_{N}=\left\{\left(\alpha_{1}, x_{1}, v_{1}\right), \ldots,\left(\alpha_{N}, x_{N}, v_{N}\right)\right\}, x_{i} \in \Omega$, $v_{i} \in \mathbb{R}^{3}$ be a particle approximation of $\bar{f} d v d x$. Then we construct an approximation of the product measure $\kappa \otimes \mu_{*} \otimes \mu \otimes \eta$ and evaluate (13) for the discrete product measure. The simulation scheme hence is:

1. For any particle $x_{i}$ choose a collision parameter $\eta_{i}$ uniformely distributed in $S^{2}$.

2. Choose uniformely a test collision partner $x_{i^{*}}$ which is situated in the support of $\beta_{h}\left(x_{i}, .+a \eta_{i}\right)$.

3. Choose a uniformely distributed $\kappa_{i} \in[0,1]$. 
4. Determine the post-collisional state $(2)$ of $\left(x_{i}, v_{i}\right)$ and $\left(x_{i^{*}}, v_{i^{*}}\right)$ at time $(k+1) \Delta t:$

$$
\left(v_{i}^{k+1}, v_{i^{*}}^{k+1}\right)= \begin{cases}\left(\left(v_{i}^{k}\right)^{\prime},\left(v_{i^{*}}^{k}\right)^{\prime}\right) & \text { if } \kappa_{i} \leq \Delta t \tilde{q}_{21} \\ \left(v_{i}^{k}, v_{i^{*}}^{k}\right) & \text { otherwise }\end{cases}
$$

5. Repeat until no more test collision pairs can be found.

Applying the two steps above for each time step $\Delta t$ a solution scheme for the inhomogeneous and instationary Enskog equation arises.

\section{Remark 3.2.}

1. For the rarefied gas case, where the Enskog equation reduces to the Boltzmann equation, a convergence proof for Nanbu's method can be found in Babovsky \& Illner [2].

2. As every single collision conserves momentum and energy, these moments are globally conserved in the scheme.

In the following section this algorithm is applied to the one-dimensional Riemann problem, where shock-wave solutions appear.

\section{Application of the Scheme to the 1d- Riemann Problem}

In the present section the propagation of a plane shock wave of a dense gas is studied. Attention is focused on the calculation of the fully formed shock profile, which appears as stationary to an observer moving with the shock front. The $x$-direction corresponds to the $e^{1}$-direction in cartesian

coordinates. It is assumed that far from the shock equilibrium conditions exist, i.e.

$$
\begin{aligned}
& \lim _{x \rightarrow-\infty} f(x, v)=\frac{n_{1}}{\left(2 \pi R T_{1}\right)^{3 / 2}} \exp \left(-\frac{\left(v-u_{1} e^{1}\right)^{2}}{2 R T_{1}}\right) \\
& \lim _{x \rightarrow+\infty} f(x, v)=\frac{n_{2}}{\left(2 \pi R T_{2}\right)^{3 / 2}} \exp \left(-\frac{\left(v-u_{2} e^{1}\right)^{2}}{2 R T_{2}}\right)
\end{aligned}
$$

and $x, n_{i}, T_{i}, u_{i} \in \mathbb{R}^{1}$ and $v, e^{1} \in \mathbb{R}^{3}$. The parameters of the downstream and upstream equilibrium states are connected by the following Rankine- 


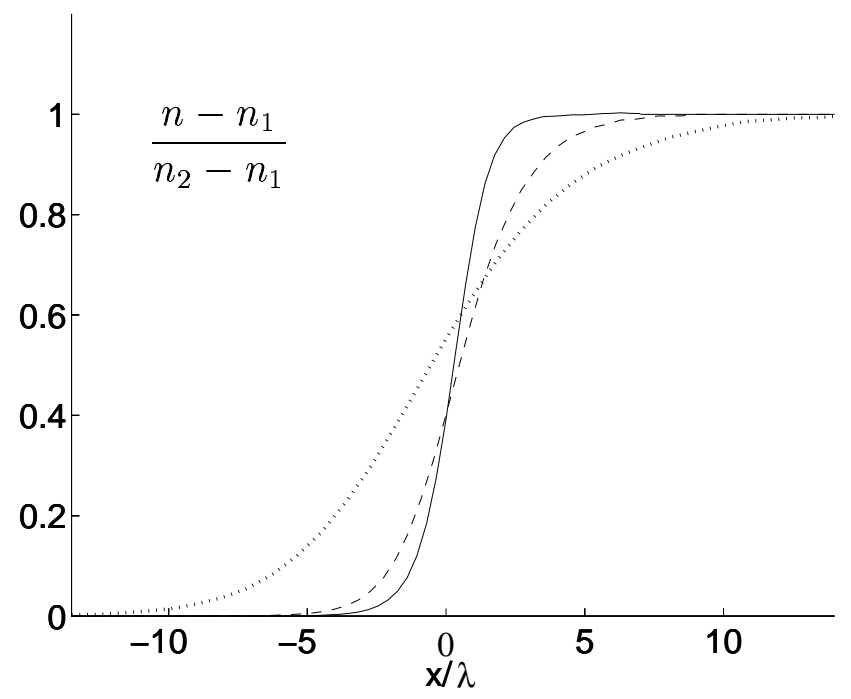

Figure 1: Normalized density profile for $M=4$ and varying $\nu$ : Solid line, $\nu=10^{-10}$. Dashed line, $\nu=0.1$. Dotted line, $\nu=0.2$.

Hugoniot relationships:

$$
\begin{aligned}
n_{1} u_{1} & =n_{2} u_{2} \\
n_{1}\left[u_{1}^{2}+R T_{1}\left(1+b n_{1} g^{(2)}\left(n_{1}\right)\right)\right] & =n_{2}\left[u_{2}^{2}+R T_{2}\left(1+b n_{2} g^{(2)}\left(n_{2}\right)\right)\right] \\
u_{1}^{2}+R T_{1}\left(5+2 b n_{1} g^{(2)}\left(n_{1}\right)\right) & =u_{2}^{2}+R T_{2}\left(5+2 b n_{2} g^{(2)}\left(n_{2}\right)\right)
\end{aligned}
$$

which arise from the equality of upstream and downstream fluxes of mass, momentum and energy. Due to the influence of the correlation function $g^{(2)}$ in (25), the ratio $n_{1} / n_{2}$ is, for constant upstream bulk velocity $u_{1}$, now varying with the reduced density $\nu$, which is not the case for a rarefied gas. The upstream mean velocity is normalized: $M=u_{1} /\left(\gamma R T_{1}\right)^{1 / 2}(\gamma=5 / 3)$. It should be noted that $M$ is not the effective Mach number, because the adiabatic speed of sound depends on $\nu$, too.

\subsection{Description of the Numerical Results}

Each computation started with the gas filling the half-space $x<0$ in the upstream equilibrium condition and the corresponding downstream equilibrium condition in the half-space $x>0$. At the end of the instationary part of the system's evolution, an averaging over many time-steps took place to smoothen the oscillations of the discrete measures.

Computational parameters such as number of particles, resolution of 


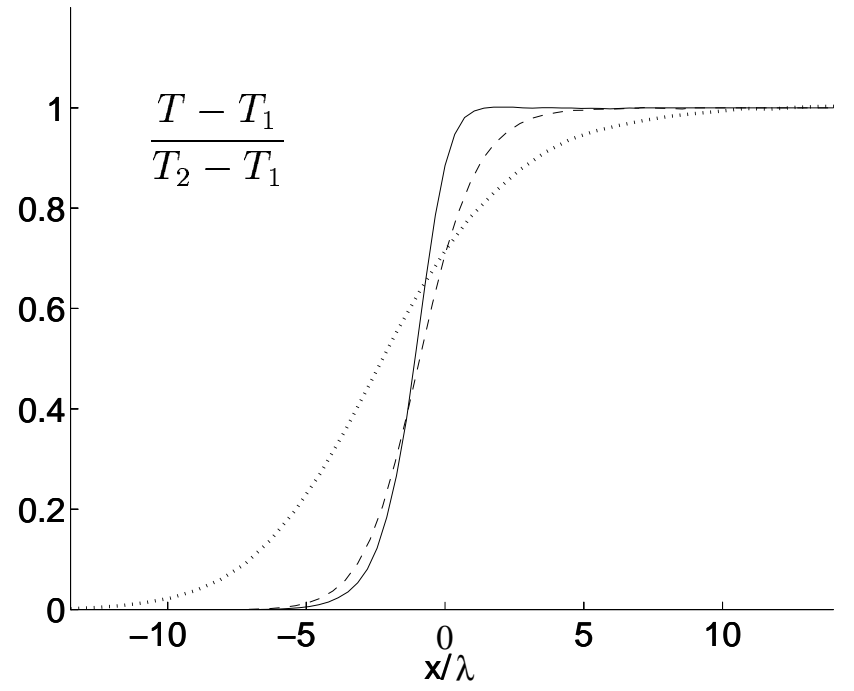

Figure 2: Normalized temperature profile for $M=4$ and varying $\nu$ : Solid line, $\nu=10^{-10}$. Dashed line, $\nu=0.1$. Dotted line, $\nu=0.2$.

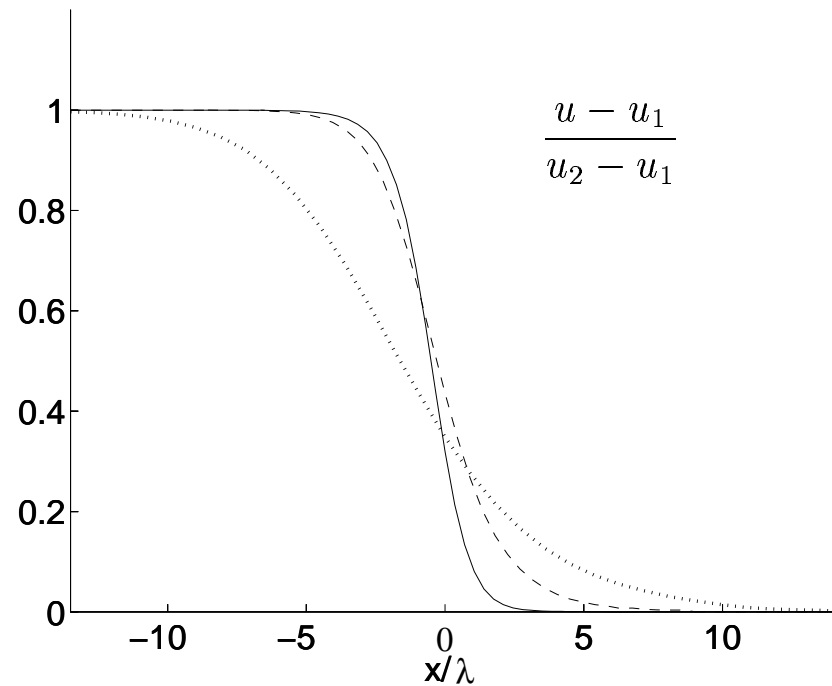

Figure 3: Normalized velocity profile for $M=4$ and varying $\nu$ : Solid line, $\nu=10^{-10}$. Dashed line, $\nu=0.1$. Dotted line, $\nu=0.2$. 
space, support of the mollifier and number of independent runs were varied until no appreciable changes in the solution were observed.

A first series of calculations considered the changing of the shock-profile for a constant upstream velocity but varying density. The result is presented in Figures $1-3$, showing the density, temperature and velocity plots of the shock-wave solution. To help comparing shock profiles in different conditions, each quantity is normalized to its own variation across the shock region. As is easily seen, the width of the shock is growing with increasing $\nu$. Even in a scaling to a constant length $\lambda_{B}=\left(\sqrt{2} \pi n a^{3}\right)^{-1}$, which is not pictured here, the widening of the shock is noticable. As in the rarefied gas case, the temperature profile precedes the density profile.

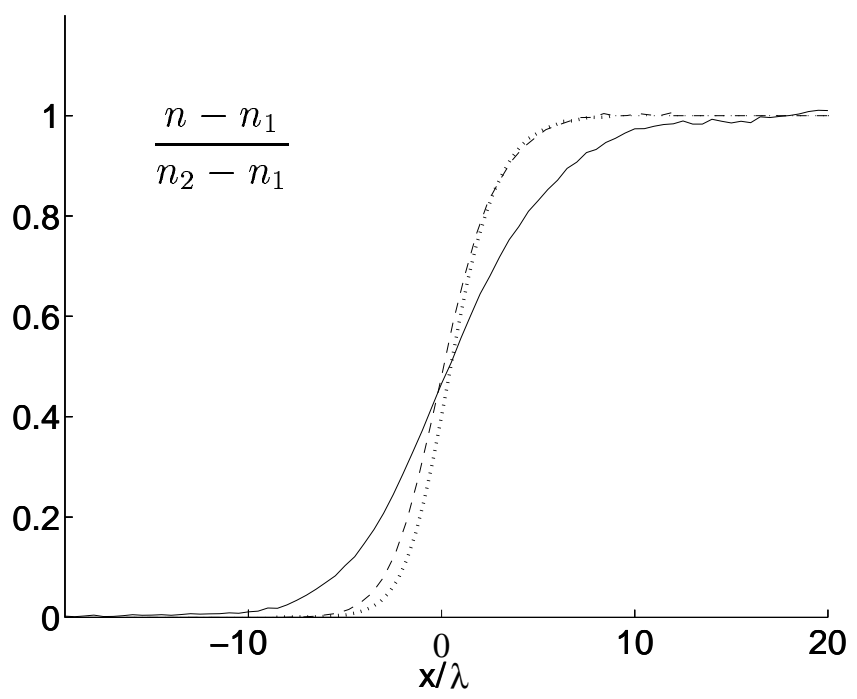

Figure 4: Normalized density profile for $\nu=0.1$ and varying $M$ : Solid line, $M=2$. Dashed line, $M=3$. Dotted line, $M=4$.

A second interest was taken in the dependency of the shock-profile on the upstream velocity if $\nu$ was fixed. From the theory of rarefied gases it is well-known that for small shock-speeds the maximum gradient of the profiles is increasing with the shock-speed, see e.g. Mott-Smith [11]. The same phenomenon appears to be true in the dense gas limit as well. In Figure 4 the reduced density was taken as $\nu=0.1$ and the shock-velocity varied.

Further calculations have been made to allow a comparison to already known results. For this the concept of the reciprocal shock thickness $\delta^{-1}$ is used, which is defined as the maximum value of the normalized density 


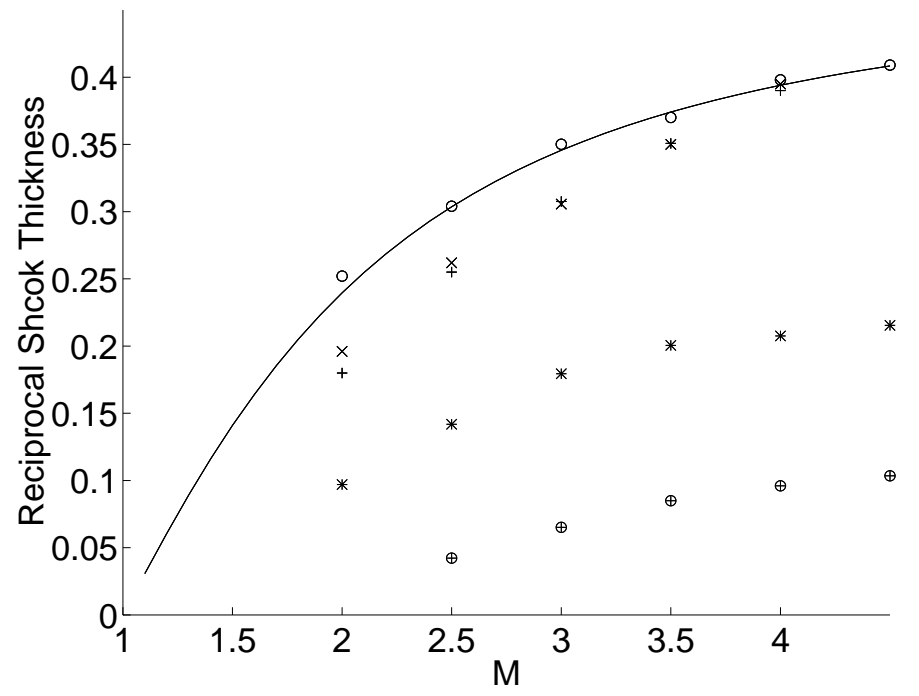

Figure 5: Reciprocal shock thickness as a function of $M$ and $\nu$. The solid line represents Mott-Smith $v^{2}$ curve for a hard-sphere potential. The circles (o) are for $\nu=10^{-10}$, the xes $(\times)$ for $\nu=0.04$ using Frezzotti's $g^{(2)}$, the pluses $(+)$ are Frezzottis results, the stars $(*)$ for $\nu=0.1$ and the opluses $(\oplus)$ for $\nu=0.2$.

gradient:

$$
\delta^{-1}=\frac{1}{n_{2}-n_{1}} \max \left(\frac{d n}{d x}\right)
$$

The data displayed in Figure 5 have also been obtained by a time average of many time steps after the initial discontinuity had changed into a stationary profile. From the averaged profile the maximum density gradient was extracted. In the rarefied limit $\nu \rightarrow 0$ the values predicted by MottSmith [11] were recovered quite well but for $M=2$.

In a further series we checked with Frezzotti's results obtained from a different pair correlation function, see [7], where in his notation $E=0.4$ was taken. This corresponds to a slightly dense gas with $\nu=0.04$. Also here the comparison is rather good for any calculated state but for small $M$.

For small $\nu$ the data is quite close to the rarefied limit, which should be expected. It also seems to converge to the low density limit with increasing shock speed, at least for the tested value $\nu=0.04$. For bigger $\nu$ this becomes more and more untrue, the shock profile widens and thus the reciprocal shock thickness decreases. 


\section{Concluding Remarks}

In this paper a finite pointset method was presented which is able to handle the inhomogeneous Enskog equation for dense gases as well as for rarefied gases. The algorithm is based on the well-known finite pointset method and conserves energy and momentum. Further, it is open to either a grid-based scheme or a grid-less one, where for the examples a grid-less mollifier was taken. It is able to calculate the profile of shock waves even if the mean free path $\lambda$ is smaller than the diameter of the molecules, e.g. for $\nu=0.2$ the diameter was $a=2.3 \lambda$. The comparison to Frezzotti's results showed a rather good agreement.

The standard Enskog theory was used hereby to model the pair correlation function $g^{(2)}$. Here it would be interesting to observe the influence of

replacing $g^{(2)}$ by the functional expression as derived by the revised Enskog theory [14], which means a higher computational effort.

\section{References}

[1] B. J. Alder and W. G. Hoover. Numerical statistical mechanics. Physics of Simple Liquids, pages 79-114, 1968. North-Holland.

[2] H. Babovsky and R. Illner. A convergence proof for Nanbu's simulation method for the full Boltzmann equation. SIAM J. Numer. Anal., 26(1):45-65, 1989.

[3] G. A. Bird. Molecular Gas Dynamics and the Direct Simulation of Gas Flows. Clarendon Press, Oxford, 1994.

[4] N. F. Carnahan and K. E. Starling. Equation of state for nonattracting rigid spheres. J. Chem. Phys., 51(2):635-636, 1969.

[5] D. Enskog. Kinetische Theorie. Svenska Akad., 63, 1921.

[6] A. Frezzotti. A particle scheme for the numerical solution of the Enskog equation. Physics of Fluids, 1996. to appear.

[7] A. Frezzotti and C. Sgarra. Numerical analysis of a shock-wave solution of the Enskog equation obtained via a Monte Carlo method. J. Stat. Phys., 73(1/2):193-207, 1993.

[8] A. Goldshtein, M. Shapiro, and C. Gutfinger. Mechanics of collisional motion of granular materials. Part 3. Self-similar shock wave propagation. J. Fluid Mech., 316:29-51, 1996. 
[9] J. M. Montanero and A. Santos. Monte Carlo simulation method for the Enskog equation. Phys. Rev. E, 54(1):438-444, 1996.

[10] D. Morgenstern. Analytical studies related to the Maxwell-Boltzmann equation. J. Rat. Mech. Anal., 4:533-555, 1955.

[11] H. M. Mott-Smith. The solution of the Boltzmann equation for a shock wave. Physics Review, 82(6):885-892, 1951.

[12] K. Nanbu. Direct simulation scheme derived from the Boltzmann equation. J. Phys. Japan, 49:2042-2049, 1980.

[13] H. Neunzert and J. Struckmeier. Boltzmann simulation by particle methods. In International Symposium on 'Boltzmann's Legacy 150 Years after his Birth'. Accademia Nazionale dei Lincei, 1997. to appear.

[14] P. Résibois. H-theorem for the (modified) nonlinear Enskog equation. J. Stat. Phys., 19(6):593-609, 1978.

[15] K. Steiner. Kinetische Gleichungen zur Beschreibung verdünnter ionisierter Gase und ihre numerische Behandlung mittels gewichteter Partikelmethoden. Phd. thesis, University of Kaiserslautern, 1995.

[16] J. Struckmeier. Die Methode der finiten Punktmengen: Neue Ideen und Anregungen. Phd. thesis, University of Kaiserslautern, 1994. 